\title{
Leiomyosarcoma of the epididymis: 2 cases and review of the literature
}

\author{
Victor T.H. Yuen, BSC,; Shane D. Kirby, PhD, MD, FRCPC; ${ }^{+}$Young Chi Woo, MD, FRCSC ${ }^{\dagger}$ \\ `The University of Queensland, Mayne Medical School, Brisbane, Australia; 'Burnaby General Hospital, Burnaby, BC
}

Cite as: Can Urol Assoc J 2011;5(6):E121-E124; http://dx.doi.org/10.5489/cuai.11008

\begin{abstract}
Paratesticular leiomyosarcomas are rare. We report two cases of leiomyosarcoma of the epididymis diagnosed and treated in a regional community hospital in Canada in 2009 and 2010. Both patients were males of ages 58 and 75, respectively. They presented with painless masses which, on ultrasonography, appeared to be solid masses demonstrating blood flow. Each patient underwent trans-scrotal resection of the affected epididymis. Pathological examination of trans-scrotal epididymectomy specimens confirmed the diagnosis of high-grade leiomyosarcoma. Leiomyosarcoma is an important differential diagnosis for paratesticular masses in the elderly male; the primary treatment is radical inguinal orchiectomy with high ligation of the spermatic cord.
\end{abstract}

\section{Introduction}

We report two cases of leiomyosarcoma of the epididymis diagnosed and treated in a regional community hospital in Canada in 2009 and 2010.

\section{Case 1}

An otherwise healthy 58-year-old man presented with a 4-year history of left testicular discomfort, associated with a sensation of friction particularly while riding his bicycle. Over the previous 6 months, he had noticed a discrete mass increasing in size. Physical examination demonstrated a palpable mass in the region of the left epididymis. Scrotal ultrasonography showed a solid, heterogeneous extra-testicular mass arising from the head of the left epididymis. The sonogram demonstrated blood flow in the mass with no cystic changes.

After discussion of the initial workup, the patient agreed to have the mass resected under general anesthesia. Using a trans-scrotal approach, we were able to free the left epididy- mis from the testicle and surrounding tissue and completely resect it.

Macroscopically, the gross specimen measuring 3.5 $\times 2.0 \times 1.0 \mathrm{~cm}$ appeared pinkish tan and much of its surface showed a yellowish lobulated lesion measuring $2.5 \mathrm{~cm}$ at widest diameter. On cut section, the tissue was homogeneous white and nodular. The tumour demonstrated several irregular nodules around a main lesion. Microscopically, the mass showed a markedly atypical mitotically active spindle cell neoplasm showing fascicular architecture extending to the resection margin with somewhat indistinct cellular outlines (Fig. 1). Mitotic rate was variable but measured up to five mitotic figures per high power field. Foci of cytologic atypia were present, with tumour-type giant cells visible (Fig. 2). The French Federation of Cancer Centers Sarcoma Group grading system score was 4 out of 8 (sum of 2 for degree of differentiation, 0 for absence of necrosis and 2 for degree of mitotic activity) which corresponds to an overall Grade 2 out of 3 . Immunohistochemical stains for desmin, smooth muscle actin and muscle specific actin were all strongly positive (Fig. 3). Stains for S-100 and c-kit were negative. These findings were diagnostic of high-grade leiomyosarcoma.

Postoperative staging computed tomography (CT) scan of the chest, abdomen and pelvis showed no evidence of metastatic disease but, because of the finding of positive margin, radical inguinal orchiectomy was subsequently performed within 3 weeks of the first surgery. The left testicle and its spermatic cord were resected en bloc to the internal inguinal ring, removing as much of the spermatic cord as possible. Pathological examination of the orchiectomy specimen showed no evidence of residual malignancy.

The patient was followed with ongoing chest x-rays every 4 months and CT imaging of the abdomen and pelvis every 6 months over the last 2.5 years, with no evidence of recurrent disease to date. 
Yuen et al.

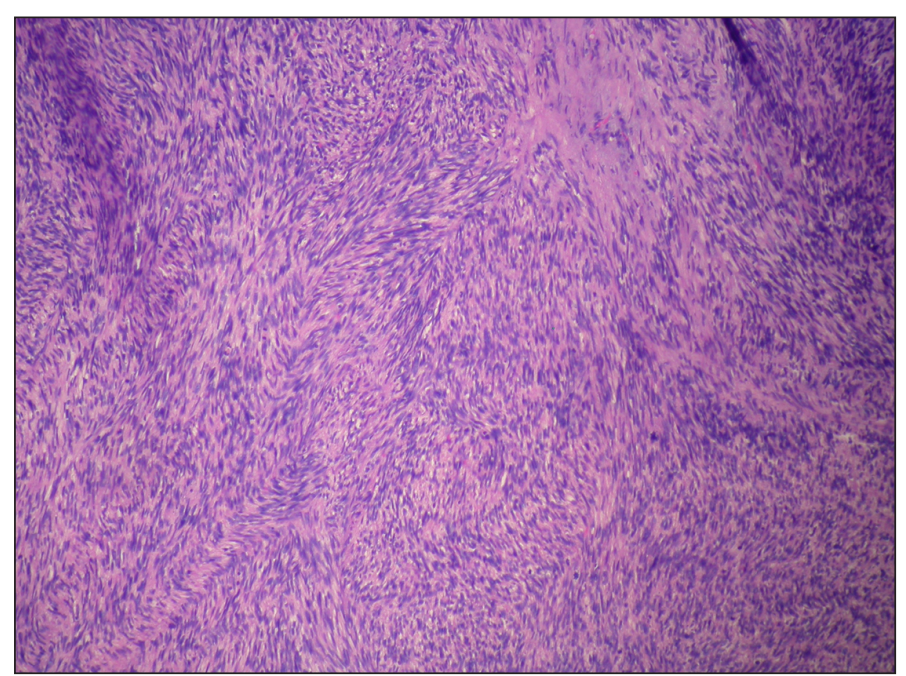

Fig. 1. Case $1 \mathrm{H} \& \mathrm{E}$ stain showing fascicular pattern of spindle cells.

\section{Case 2}

A 75-year-old male with a history of minor urethral bleeding presented for assessment. On physical examination, a painless mass was palpable in the region of the right epididymis. Scrotal ultrasonography showed an enlarged hypoechoic, heterogeneous 3.8-cm vascular epididymal mass.

The patient agreed to trans-scrotal resection of the epididymal under general anesthesia. He tolerated the procedure well, but developed a local hematoma which persisted postoperatively for 2 weeks.

The resected specimen measuring $4.5 \times 3.5 \times 3.0 \mathrm{~cm}$ was firm and appeared pinkish tan on gross inspection. On cut section, it consisted of a vaguely lobulated, fleshy appearing tan lesion. Histologically, this tumour showed a pleomorphic mitotically active spindle cell neoplasm with fascicular architecture extending to the resection margin (Fig. 4). Cytoplasmic outlines were poorly defined and multinucleated cells were identified in some areas. Mitotic rate was about 14 mitotic figures per ten high power fields (Fig. 5). Therefore, the French score was 4 (sum of 2 for differentiation, 0 for necrosis and 2 for mitotic activity) corresponding to Grade 2 as per the previous case. Immunohistochemical stains confirmed the tumour as strongly positive for vimentin, desmin, muscle specific actin and smooth muscle actin (Fig. 6). These histologic features were again diagnostic of high-grade leiomyosarcoma.

Three weeks after the first surgery, radical inguinal orchiectomy with high ligation of the spermatic cord to the level of internal ring was performed. During the procedure, the right testicle was observed to have become firmly adhered to the scrotal wall, most likely due to postoperative scar tissue formation. Because of concerns of residual tumour in the wound, part of the right scrotum was resected en bloc with the testicle and spermatic cord. Subsequent microscopic

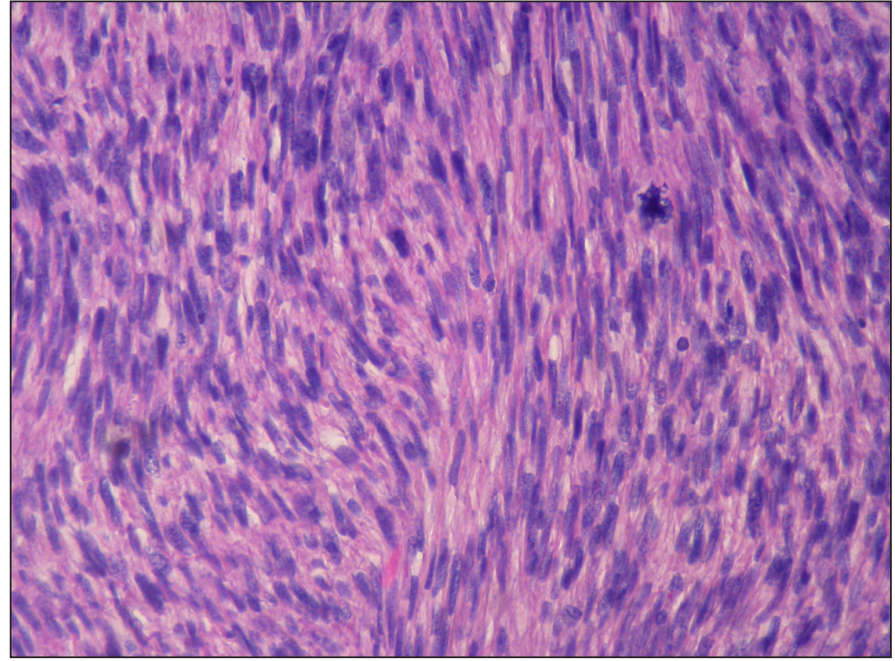

Fig. 2. Case $1 \mathrm{H}$ \& E stain showing mitotic activity and atypical nuclei.

examination of the orchiectomy specimen showed no evidence of residual disease.

The patient was referred to the local cancer clinic for ongoing follow-up with regular chest x-rays and CT scans of the abdomen and pelvis. No evidence of lymphadenopathy was reported from the first set of CT scans. He has remained free of disease for 5 months since the initial epididymectomy.

\section{Discussion}

Paratesticular sarcomas are rare. As such, it is difficult even for large institutions to accumulate sufficient cases to document the natural history of these tumour s and draw conclusions about treatment results. ${ }^{1}$ Paratesticular sarcomas fall under the category of soft tissue sarcomas. In the United States, 9500 new cases of soft tissue sarcoma were diagnosed in $2006,{ }^{2}$ which is less than $1 \%$ of all new cancer cases. In Canada, 1050 new cases of soft tissue sarcoma were diagnosed in the same year. ${ }^{3}$ According to a large case series from the Memorial Sloan-Kettering Cancer Center, only $2.1 \%$ of soft tissue sarcomas arise in the genitourinary tract, ${ }^{4}$ of which $44 \%$ are paratesticular. ${ }^{5}$

Leiomyosarcoma is the most commonly reported histologic type of paratesticular sarcoma. ${ }^{6}$ Peak incidence is reported to occur in the sixth and seventh decades of life, ${ }^{6}$ as seen with our cases. Earlier literature similarly reported that the average age for paratesticular leiomyosarcoma was 60 and most cases were in the 40 to 70 age group. $^{7}$ Leiomyosarcomas of the epididymis are less common than those of the spermatic cord. ${ }^{6}$

Given these statistics, the finding of two cases of leiomyosarcoma of the epididymis in a small regional hospital in two consecutive years was unusual.

Leiomyosarcoma is thought to originate from smooth muscle cells of mesenchymal origin in the wall of epididy- 


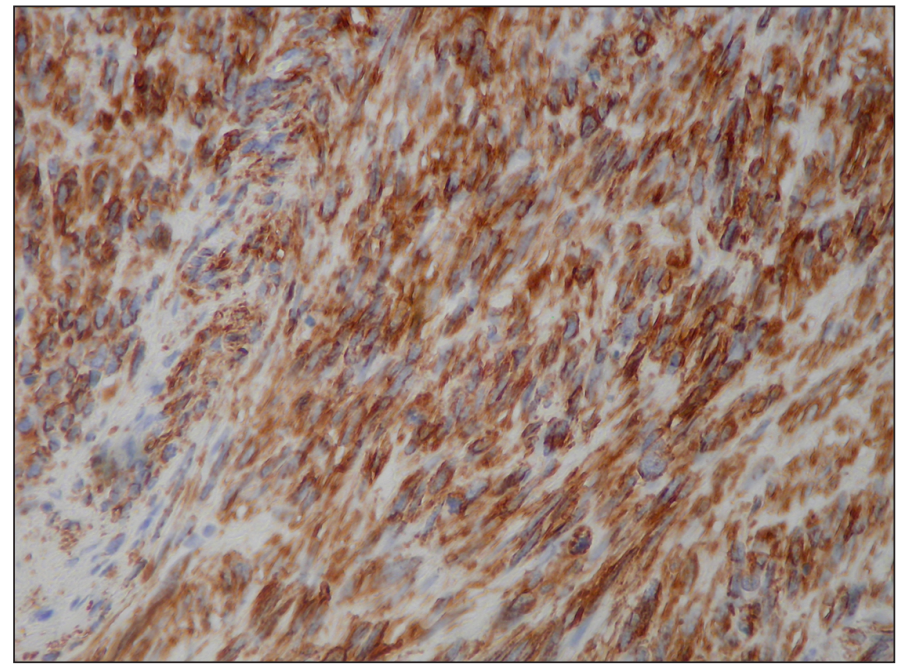

Fig. 3. Case 1 Positive desmin immunohistochemistry.

mal tubule or its vasculature. Like other sarcomas, it tends to infiltrate local tissues. ${ }^{8}$ Epididymal leiomyosarcoma metastasizes via lymphatic drainage to the retroperitoneal nodes and hematogenously to the lungs.

Paratesticular leiomyosarcoma is difficult to diagnose preoperatively, usually presenting as a painless, firm mass that may cause discomfort as it impinges on nearby structures. On palpation, the mass is usually well-defined and lobulated, easily mobile within the scrotum and sometimes associated with a small hydrocele. ${ }^{7}$ Investigation should begin with scrotal ultrasonography to determine the size and location of the mass, as well as to characterize its density and vascularity. Paratesticular tumour appears on a sonogram as a solid mass with blood flow, but an inflammatory process like epididymitis can have similar appearance. Moreover, epididymitis is common. According to the Nickel and colleagues in the Canadian PIE prostatitis, interstitial cystitis, and epididymitis (PIE) study, ${ }^{9}$ about $1 \%$ of men seen in an outpatient urologic practice are diagnosed with epididymitis. Therefore, given the relative rareness of paratesticular malignancy, the choice of going directly to radical inguinal orchiectomy rather than trans-scrotal epididymectomy seems debatable.

Definitive diagnosis of leiomyosarcoma requires histologic examination of a resected specimen for features of smooth muscle differentiation and malignancy. In addition, differential diagnoses of a reactive process, such as inflammatory pseudotumour or benign neoplasia such as leiomyoma or schwannoma, must be ruled out. Grossly, leiomyosarcomas present as fleshy mass lesions. ${ }^{10}$ The classic histologic features are that of a cellular pleomorphic spindle cell neoplasm with fascicular architecture at low power, at high power showing eosinophilic cytoplasm containing delicate longitudinal fibrils and blunt-ended nuclei. ${ }^{11,12}$ Foci of cytologic atypia, such as abnormal cruciform mitoses and giant cells, may be present. Necrosis may be seen in high-grade tumours. Smooth muscle differentiation is exemplified by an

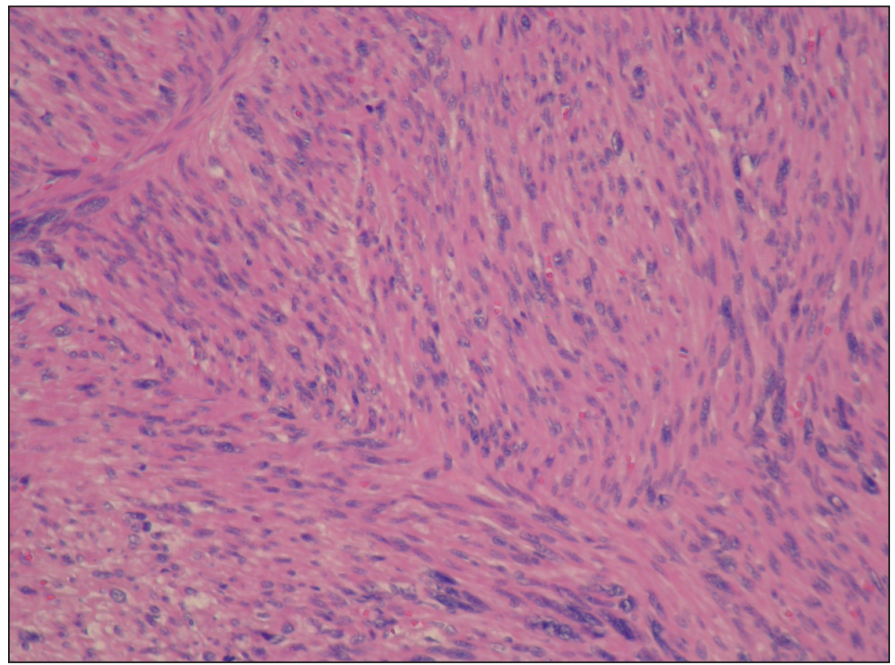

Fig. 4. Case $2 \mathrm{H}$ \& E stain showing fascicular pattern of spindle cells.

immunohistochemical profile that is positive for expression of smooth muscle actin, muscle-specific actin and desmin. ${ }^{1}$

Additionally, h-caldesmon may be used to confirm smooth muscle differentiation and myogenin to exclude spindle cell rhabdomyosarcoma, which can express smooth muscle actin. ${ }^{13}$ Positive staining for CD34 may be seen on endothelial cells of vessels supplying the tumour. ${ }^{14}$ High mitotic activity is an important criterion for differentiating leiomyosarcoma from leiomyoma. However, the threshold number of mitotic figures per high power field that characterizes malignant growth varies depending on the anatomic site from which the tumour arises. For a deep-seated smooth muscle tumour in the paratesticular region, we consider any mitotic activity with nuclear atypia to be a marker of malignancy. ${ }^{15}$

The clinical and biologic behavior of paratesticular leiomyosarcoma is difficult to predict. However, an authoritative review suggested that the natural course of disease depends on site, size, grade and evidence of nodal or distant metastasis. ${ }^{15}$ While low-grade tumours behave indolently, high-grade tumours are aggressive and associated with poor clinical outcome. ${ }^{1}$ In a retrospective study of 14 cases, 5 of 8 patients with high-grade tumours had systemic failure or spread to retroperitoneal nodes. ${ }^{16}$

There is relatively little evidence-based consensus on the management of paratesticular leiomyosarcoma. Radical orchiectomy is the accepted primary treatment, ${ }^{17}$ but there remains the problem of local recurrence. Complete surgical excision can achieve negative margins, but wide resection margins can be difficult to obtain in the paratesticular region. With local excision and surveillance, the survival rate has been reported to be around $50 \%$ to $80 \%$ and there is microscopic residual disease in $27 \%$ of cases that underwent repeat excision. ${ }^{18}$ Others have reported that one-third of patients were harboring occult local residual disease following local excision or orchiectomy. ${ }^{16}$ Given 
Yuen et al.

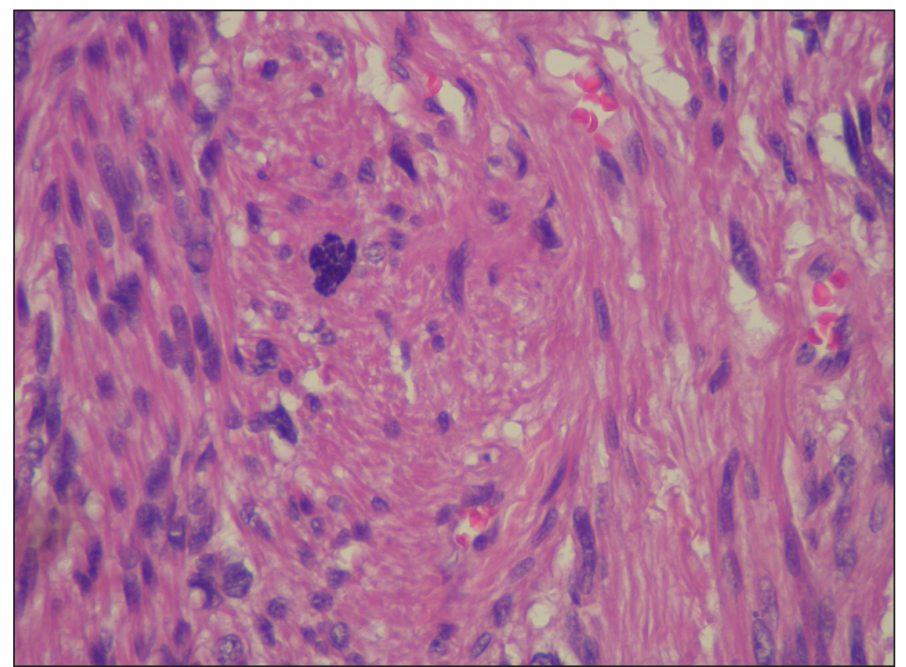

Fig. 5. Case 2 H \& E stain showing mitotic activity and atypical nuclei.

these reported rates of recurrence, there is a clear need for long-term follow-up. Regular chest $\mathrm{x}$-rays and abdominopelvic CT scans are recommended.

Adjuvant therapy may help limit local recurrence but options tend to be limited. Retroperitoneal lymph node dissection has no significant survival benefits ${ }^{19}$ and is difficult to justify. A frequently-cited study involving 18 patients demonstrated some benefit of radiation therapy, ${ }^{20}$ but radiation was not indicated for our cases because negative margins were achieved with repeat resection. Fagundes and colleagues found local recurrence in 5 of 9 patients who had orchiectomy alone, but there was no recurrence in the other group of 9 patients who had received adjuvant radiation therapy. In terms of systemic therapy, doxorubicin-based adjuvant chemotherapy has been shown in a meta-analysis to be somewhat effective for soft tissue sarcomas at various sites, but statistically significant improvement in overall survival could not be shown. ${ }^{21}$

\section{Conclusion}

Leiomyosarcoma is an important differential diagnosis for paratesticular masses in the elderly male. The primary management is radical inguinal orchiectomy with high ligation of the spermatic cord and subsequent long-term follow-up.

Competing interests: None declared.

This paper has been peer-reviewed.

\section{References}

1. Fisher C, Goldblum JR, Epstein JI, et al. Leiomyosarcoma of the paratesticular region: a clinicopathologic study. Am I Surg Pathol 2001;25:1143-9.

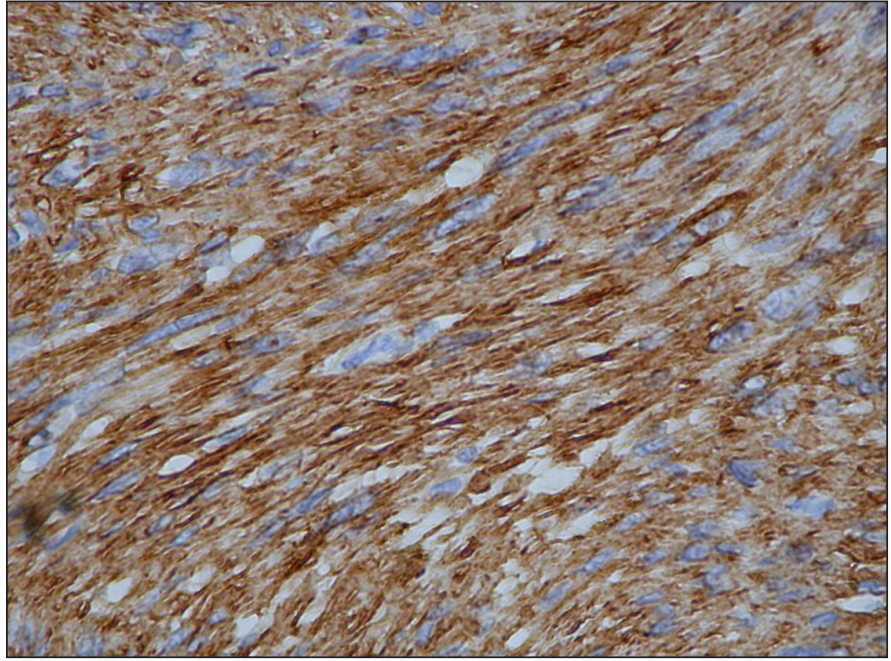

Fig. 6. Case 2 Positive desmin immunohistochemistry.

2. Ries LAG, Harkins D, Krapcho M, et al. SEER Cancer Statistics Review, 1975-2003. Bethesda, MD: National Cancer Institute 2006.

3. Canadian Cancer Statistics 2010. Toronto, ON: Canadian Cancer Society 2010.

4. Stojadinovic A, Leung DH, Allen P, et al. Primary adult soft tissue sarcoma: time-dependent influence of prognostic variables. I Clin Oncol 2002;20:4344-52.

5. Dotan ZA, Tal R, Golijanin D, et al. Adult genitourinary sarcoma: the 25-year Memorial Sloan-Kettering experience. J Urol 2006;176:2033-8; discussion 2038-9.

6. Weiss SW, Goldblum JR. Enzinger and Weiss's soft tissue tumour s. 4th ed. St. Louis, Ml: Mosby; 2001.

7. Rushworth GF. Leiomyosarcoma of the epididymis. Proc R Soc Med 1971;64:999.

8. Varzaneh FE, Verghese M, Shmookler BM. Paratesticular leiomyosarcoma in an elderly man. Urology 2002;60:1112.

9. Nickel JC, Teichman JM, Gregoire $M$, et al. Prevalence, diagnosis, characterization, and treatment of prostatitis, interstitial cysitits, and epididymitis in outpatient urological practice: the Canadian PIE Study. Urology 2005; 66:935-40.

10. Evans HL, Shipley J. Pathology and Genetics of Tumours of Soft Tissue and Bone. Lyon: World Health Organization 2002.

11. Hashimoto H, Daimaru Y, Tsuneyoshi M, et al. Leiomyosarcoma of the external soft tissues. A clinicopathologic, immunohistochemical, and electron microscopic study. Cancer 1986;57:2077-88.

12. Wile AG, Evans HL, Romsdahl MM. Leiomyosarcoma of soft tissue: a clinicopathologic study. Cancer 1981;:48:1022-32.

13. Stock N, Chibon F, Binh MB, et al. Adult-type rhabdomyosarcoma: analysis of 57 cases with clinicopathologic description, identification of 3 morphologic patterns and prognosis. Am I Surg Pathol 2009;33:1850-9.

14. Silvestri F, Banavali S, Baccarani $M$, et al. The CD34 hemopoietic progenitor cell associated antigen: biology and clinical applications. Haematologica 1992;77:265-73.

15. Folpe AL, Weiss SW. Paratesticular soft tissue neoplasms. Semin Diagn Pathol 2000;17:307-18.

16. Catton C, Jewett $M, O^{\prime}$ Sullivan $B$, et al. Paratesticular sarcoma: failure patterns after definitive local therapy. J Urol 1999;161:1844-7.

17. Planz B, Brunner K, Kalem T, et al. Primary leiomyosarcoma of the epididymis and late recurrence on the penis. J Urol 1998; 159:508.

18. Blitzer PH, Dosoretz DE, Proppe KH, et al. Treatment of malignant tumour s of the spermatic cord: a study of 10 cases and a review of the literature. J Urol 1981;126:611-4.

19. Banowsky LH, Shultz GN. Sarcoma of the spermatic cord and tunics: review of the literature, case report and discussion of the role of retroperitoneal lymph node dissection. J Urol 1970;103:628-31.

20. Fagundes MA, Zietman AL, Althausen AF, et al. The management of spermatic cord sarcoma. Cancer 1996;77:1873-6.

21. Tierney JF, Stewart LA, Parmar MKB. Adjuvant chemotherapy for localised resectable soft-issue sarcoma of adults: meta-analysis of individual data. Lancet 1997;350:1647-54.

Correspondence: Dr. Victor Tsun Ho Yuen, The University of Queensland, Mayne Medical School, 288 Herston Rd, Brisbane, QLD, 4006 Australia; victor.yuen@uqconnect.edu.au 